\title{
DIFFEOMORPHIC INVARIANTS OF IMMERSED CIRCLES
}

\author{
BY
}

\author{
ROGER F. VERHEY
}

\begin{abstract}
The intersection sequences of a normal immersion form a complete invariant for diffeomorphically equivalent normal immersions. Numerical invariants and inequalities on numerical invariants are obtained using intersection sequences.
\end{abstract}

1. Introduction. A mapping $f$ from the oriented circle $S^{1}$ into the oriented plane $R^{2}$ is called a regular immersion iff $f \in C^{1}$ and $f^{\prime}(t) \neq 0$ for all $t$. An image point $f(t)$ is called a simple intersection point of $f$ when $f(t)$ has exactly two preimages with the corresponding tangent vectors being linearly independent. Whitney [6] introduced the concept of a normal immersion as a regular immersion with a finite number of simple intersection points and with all other image points having exactly one preimage. The work of Whitney [6] and Titus [4] shows that the normal immersions are generic (dense and open) in the space of regular immersions with the usual $C^{1}$-topology defined by

$$
\|f-g\|=\max _{t \in s^{1}}\left\{|f(t)-g(t)|+\left|f^{\prime}(t)-g^{\prime}(t)\right|\right\} .
$$

In the paper of Titus, a foundation is laid for the systematic study of normal immersions using relations between the simple intersection points embodied in a combinatorial tool called an intersection sequence.

In this paper we continue the study of normal immersions using the combinatorial aspects of intersection sequences. In $\$ 2$ we show that the set of distinct intersection sequences of a normal immersion is a complete invariant for diffeomorphically equivalent normal immersions. In $\$ 3$ we obtain an algorithm defined on intersection sequences for computing the winding number of a normal immersion with respect to an arbitrary point. This leads to a new proof of the fact that the maximum winding number ( $w$ max) and the minimum winding number ( $w$ min) are numerical invariants for diffeomorphically equivalent normal immersions. $\S 4$ contains the inequality $O(f) \geqq 2(w \max (f)-w \min (f))$ where $O(f)$ is the order of the normal immersion $f$. $\S 5$ contains the results on subloops of a normal immersion needed in the last two sections. In $\S 6$ we give an inductive proof

Received by the editors November 6, 1968.

AMS 1970 subject classifications. Primary 57D40; Secondary 58D10, 30A90, 30A32.

Key words and phrases. Normal immersion, intersection sequence, diffeomorphically equivalent, complete invariant, numerical invariant, winding number, tangent winding number, total variation. 
of a result due to Whitney [6] which computes the tangent winding number $\tau$ of a normal immersion $f$ from an intersection sequence of $f$. As a corollary we obtain the fact that $\tau$ is a numerical invariant. Also in $\$ 6$, we obtain the inequality $n(f)$ $\geqq 2 w \max (f)-\tau(f)-1$ where $n(f)$ is the number of simple intersection points of $f$. In $\S 7$ we show that $w \max (f) \leqq(1 / 2 \pi) v(f)$ where $v$ is the total variation of $f$. Corollaries of this are necessary conditions for a normal immersion to be simple and classical results in function theory due to Umezawa [5] and Loewner [3] concerning bounds on the valence of meromorphic functions.

2. Diffeomorphic equivalence and a complete invariant. Two normal immersions $f$ and $g$ are said to be diffeomorphically equivalent, written $f \sim g$, if there exists sense-preserving diffeomorphisms $\gamma: S^{1} \rightarrow S^{1}$ and $\Gamma: R^{2} \rightarrow R^{2}$ such that $g=$ $\Gamma \circ f \circ \gamma$ on $S^{1}$. A mapping $\mathscr{F}$ defined on the space of normal immersions is said to be an invariant if $f \sim g$ implies that $\mathscr{F}(f)=\mathscr{F}(g)$. An invariant mapping $\mathscr{F}$ is said to be a complete invariant if $\mathscr{F}(f)=\mathscr{F}(g)$ implies that $f \sim g$. The purpose of this section is to demonstrate the following:

THEOREM 2.1. The set of intersection sequences of a normal immersion is a complete invariant.

Before proving this statement we shall make it precise by defining the intersection sequences of a normal immersion. Let $f$ be a normal immersion represented as the complex-valued function $f(t)=f_{1}(t)+i f_{2}(t)$. Let $[f]$ denote the oriented image of $S^{1}$ under $f$. The outer boundary of $f$ is the boundary of the unbounded component of the complement of $[f]$. Let $n$ be the number of simple intersection points of $f$ and fix $t_{0}$ such that $f\left(t_{0}\right)$ is on the outer boundary and is not a simple intersection point. Since each simple intersection point has two distinct preimages, there are $2 n$ parameter values of $f$ corresponding to the $n$ simple intersection points. Let $x_{1}, x_{2}, \ldots, x_{2 n}$ be these parameter values with $t_{0}=x_{0}<x_{1}<\cdots<x_{2 n}<x_{2 n+1}$ $=t_{0}+2 \pi$. The intersection sequence of $f$ with respect to $t_{0}$, denoted by $\operatorname{IS}\left(f, t_{0}\right)^{6}$ consists of

(1) the integers 0 through $2 n+1$,

(2) a fixed point free involution * defined on the integers 0 through $2 n+1$ by $0^{*}=2 n+1$ and $i^{*}=j$ iff $f\left(x_{i}\right)=f\left(x_{j}\right)$, and

(3) a signing of the integers 0 through $2 n+1$, denoted by $\nu(j)$, defined by $\nu(j)=\operatorname{sgn} \operatorname{det}\left[f^{\prime}\left(x_{i}\right), f^{\prime}\left(x_{j}\right)\right]$ where $i=j^{*}$ for $j=1, \ldots, 2 n$ and with $\nu(0)=-\nu(2 n+1)$ being the sign of the determinant of the outward normal at $f\left(t_{0}\right)$ with $f^{\prime}\left(t_{0}\right)$.

Normally we will write $I S$ to denote an intersection sequence of $f$ instead of the more explicit notation IS $\left({ }^{\circ} f, t_{0}\right)$. The only time we use the notation IS $\left(f, t_{0}\right)$ is when the parameter value $t_{0}$ is also used to determine an intersection sequence of a different normal immersion in the same context. Moreover, since $S^{1}$ is diffeomorphically equivalent to the interval $[0,2 n+1]$ with the endpoints identified and with the integer $i$ corresponding to the parameter $x_{i}$, we shall often assume that the domain of $f$ is $[0,2 n+1]$. 
As an example of an intersection sequence of a normal immersion consider the normal immersion represented in Figure 1.

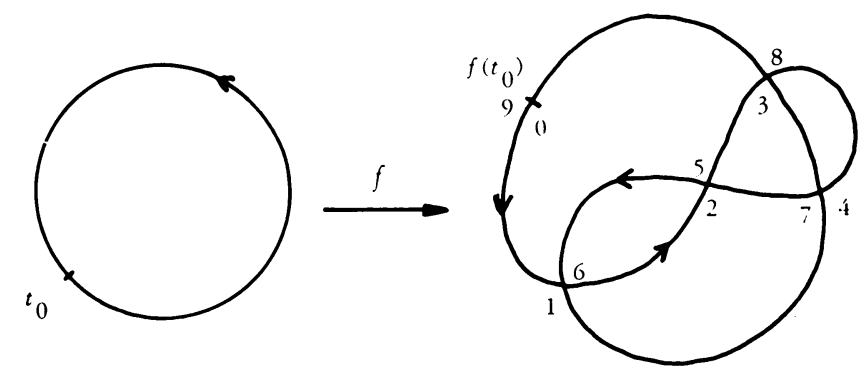

FIGURE 1

Since $f$ has four simple intersection points, IS $\left(f, t_{0}\right)$ consists of the integers 0 through 9. The involution on and the signing of these integers is obtained by traversing the oriented curve $[f]$ starting at $f\left(t_{0}\right)$ and numbering the simple intersection points consecutively. Thus $1^{*}=6,2^{*}=5,3^{*}=8,4^{*}=7,0^{*}=9$ and $\nu(0)$ $=-\nu(9)=+1, \quad \nu(1)=-\nu(6)=+1, \quad \nu(2)=-\nu(5)=-1, \quad \nu(3)=-\nu(8)=-1, \quad \nu(4)=$ $-\nu(7)=+1$.

For a given intersection sequence $I S$ of a normal immersion $f$ we define the following relations on the integers $j<j^{*}$ in $I S$ :

(1) $j \subset k$ iff $k<j<j^{*}<k^{*}$,

(2) $j L k$ iff $j<k<j^{*}<k^{*}$,

(3) $j R k$ iff $k<j<k^{*}<j^{*}$, and

(4) $j \mid k$ iff $k<k^{*}<j<j^{*}$ or $j<j^{*}<k<k^{*}$.

Let $f$ be a normal immersion and let $\rho(f)$ be the collection of distinct intersection sequences of $f$. Since the outer boundary of $f$ contains a finite number of simple intersection points and since all points on the outer boundary between two simple intersection points give rise to the same intersection sequence, we have that $\rho(f)$ is finite.

We now restate and prove Theorem 2.1.

THEOREM 2.1. $\rho$ is a complete invariant.

Proof. Suppose $f$ and $g$ are normal immersions with $\rho(f)=\rho(g)$. Let $\operatorname{IS}\left(f, t_{0}\right)$ be an intersection sequence of $f$ and let $\alpha$ be the diffeomorphism of $S^{1}$ onto $[0,2 n+1]$ determined by this intersection sequence. Since $\rho(f)=\rho(g), \operatorname{IS}\left(f, t_{0}\right)$ is also an intersection sequence of $g$ with respect to a parameter value $t_{0}^{\prime}$. That is, IS $\left(f, t_{0}\right)=$ IS $\left(g, t_{0}^{\prime}\right)$. Let $\beta$ be the diffeomorphism of $S^{1}$ onto [0, $\left.2 n+1\right]$ determined by the intersection sequence IS $\left(g, t_{0}^{\prime}\right)$. The diffeomorphism $\alpha^{-1} \circ \beta$ of $S^{1}$ into $S^{1}$ is the required diffeomorphism $\gamma$ in the definition of diffeomorphically equivalent normal immersions. The diffeomorphism $\Gamma$ of $R^{2}$ into $R^{2}$ will be constructed using the concept of a normal tubular neighborhood as defined by Francis [2, Chapter 6]. 
Let $h$ be a normal immersion of $[0,2 n+1]$ into $R^{2}$ and let $e$ be a positive real number such that $h$ is univalent on intervals of length less than $2 e$. Let $D(e)=[-e, e]$ $\times[0,2 n+1], \square_{i}=[-e, e] \times[i-e, i+e]$ for $i=1, \ldots, 2 n$, and $J(e)=D(e)-\bigcup_{i=1}^{2 n} \square_{i}$. A normal tubular neighborhood (n.t.n.) of $h$ is a $C^{1}$-immersion $H: D(e) \rightarrow R^{2}$ such that

(1) $H(0, t)=h(t)$,

(2) if $i^{*}=j$ and $r_{i j}$ is the rigid motion

$$
r_{i j}: \square_{j} \rightarrow \square_{i}:(x, j+t) \rightarrow(\nu(i) t, i+\nu(j) x),
$$

then $H_{j}=H_{i} \circ r_{i j}$ where $H_{j}=H \mid \square_{j}$, and

(3) $H$ is unipotent on $J(e)$ (each point of $H(J(e)$ ) has exactly one preimage in $D(e)$ ) and bipotent on $\square_{i}$ for $i=1, \ldots, 2 n$ (each point of $H\left(\square_{i}\right)$ has exactly two preimages in $D(e)$ ).

The existence of a n.t.n. for a normal immersion is the result of Chapter 6 of Francis [2]. There also one will find the following properties of a n.t.n. $H$ of a normal immersion $h$ listed.

(1) $H$ is an embedding of $J(e)$.

(2) $\left[H_{i}\right]$ and $\left[H_{j}\right]$ are identical or disjoint according as $i^{*}=j$ or $i^{*} \neq j$ respectively.

(3) If $e^{\prime}<e$, then $H$ restricted to $D\left(e^{\prime}\right)$ is also a n.t.n. of $h$.

(4) $H$ is univalent on any strip $[-e, e] \times[t-e, t+e]$.

(5) $H(J(e))$ is disjoint from every $\left[H_{i}\right]$.

Now let $F$ be a n.t.n. of $f$ on a domain $D\left(e_{1}\right)$ and let $G$ be a n.t.n. of $g$ on a domain $D\left(e_{2}\right)$. Select $e<\min \left(e_{1}, e_{2}\right)$. Then both $F$ and $G$ are normal tubular neighborhoods on $D(e)$. Since $F$ is unipotent on $J(e)$ and bipotent on $\square_{i}, i=1, \ldots$, $2 n$, with $F\left(\square_{i}\right)=F\left(\square_{j}\right)$ iff $i^{*}=j$, we consider the mapping $\hat{F}$ which is the restriction of $F$ to the set $J \square(e)=J(e) \cup\left(\bigcup_{i<i^{*}} \square_{i}\right)$. Then $\hat{F}$ is a diffeomorphism of $J \square(e)$ onto $[F]$. Similarly, the restriction $\hat{G}$ of $G$ to $J \square(e)$ is a diffeomorphism onto $[G]$. Thus, $\hat{G} \circ \hat{F}^{-1}$ is a diffeomorphism of $[F]$ onto $[G]$.

Assuming that $\hat{G} \circ \hat{F}^{-1}$ can be extended to a diffeomorphism of $R^{2}$ onto $R^{2}$, which we shall show presently, we now show that $\hat{G} \circ \hat{F}^{-1}$ is the required $\Gamma$ in the definition of diffeomorphically equivalent normal immersions. We work with the elements $(0, t)$ of $D(e)$ and the diffeomorphisms $\alpha$ and $\beta$ determined in the first paragraph of this proof.

(1) If $(0, t) \in J \square(e)$, then $\hat{F}(0, t)=F(0, t)=f_{\alpha}^{-1}(t)$ and $\hat{G} \hat{F}^{-1} f \alpha^{-1}(t)=\hat{G}(0, t)$ $=g \beta^{-1}(t)$.

(2) If $(0, t) \in D(e)-J \square(e)$, then $(0, t) \in \square_{i^{*}}$ for some $i<i^{*}$ and $f \alpha^{-1}(t)=F_{i} \cdot(0, t)$ $=\hat{F} r_{i i} \cdot(0, t)$.

Hence, $\hat{G} \hat{F}^{-1} f \alpha^{-1}(t)=\hat{G} r_{i i^{*}}(0, t)=G_{i} r_{i i} \cdot(0, t)=G_{i} \cdot(0, t)=g \beta^{-1}(t)$.

From (1) and (2) it follows that for all $t \in[0,2 n+1], \hat{G} \hat{F}^{-1} f \alpha^{-1}=g \beta^{-1}$. Thus, on $S^{1}, \hat{G} \hat{F}^{-1} f_{\alpha}^{-1} \beta=g$.

The diffeomorphism $\hat{G} \circ \hat{F}^{-1}$ will be extended to a diffeomorphism of $R^{2}$ into $R^{2}$ by extending it to a diffeomorphism between each of the bounded components of 
the complement of $[F]$ and the corresponding bounded component of the complement of $[G]$ and finally to a diffeomorphism between the unbounded components. Let $F_{a}$ be a bounded component of $R^{2}-[F]$ and let $r$ be a parametrization with unit tangent vector of a smooth simple closed curve in $[F]$ approximating the boundary of $F_{a}$. In addition the curve $[r]$ is to be given the interior unit normal fringe. Since $[r]$ is in $[F], \hat{G} \hat{F}^{-1}([r])$ is a smooth simple closed curve in $[G]$ approximating the boundary of the component $G_{a}$ corresponding to $F_{a}$. Moreover, the interior fringe on $[r]$ is carried into an interior fringe on $\left[\hat{G} \hat{F}^{-1} r\right]$. By Lemma 7 of Francis [2], there exist diffeomorphisms $E$ and $H$ of the disk $D^{1}$ into $R^{2}$ such that $E \mid S^{1}=r$ and $H \mid S^{1}=\hat{G} \hat{F}^{-1} r$; in addition, the radial and tangential partials of $E$ and $H$ on $S^{1}$ give the fringes and tangent vectors of $[r]$ and $\left[\hat{G} \hat{F}^{-1} r\right]$ respectively. Thus, $H \circ E^{-1}$ is a diffeomorphism of $[r] \cup \operatorname{Int}[r]$ (Int =interior) onto $\left[\hat{G} \hat{F}^{-1} r\right] \cup \operatorname{Int}\left[\hat{G} \hat{F}^{-1} r\right]$ which extends $\hat{G} \circ \hat{F}^{-1}$ to the component $F_{a}$. Thus, $\hat{G} \circ \hat{F}^{-1}$ is extendable to a diffeomorphism of $[F]$ and the bounded components of $R^{2}-[F]$ onto $[G]$ and the bounded components of $R^{2}-[G]$.

In order to finish the proof we must extend $\hat{G} \circ \hat{F}^{-1}$ to a diffeomorphism between the unbounded components of $R^{2}-[F]$ and $R^{2}-[G]$. Let $r$ be a parametrization of a smooth simple closed curve in $[F]$ approximating the boundary of the unbounded component. In this case we take the exterior unit normal fringe on $[r] . \hat{G} \circ \hat{F}^{-1}$ maps $[r]$ and its fringe onto a smooth simple closed curve $\left[\hat{G} \hat{F}^{-1} r\right]$ with an exterior fringe. By Moebius transformations we reflect $[r]$ and its fringe in a circle in the interior of $[r]$ and we reflect $\left[\hat{G} \hat{F}^{-1} r\right]$ and its fringe in a circle in its interior. In each case smooth simple closed curves with interior fringes are obtained. By applying Lemma 7 of Francis [2], we obtain a diffeomorphism $B$ between these curves and their interiors. The required diffeomorphism between $[r] \cup$ ext $[r]$ (ext $=$ exterior) and $\left[\hat{G} \hat{F}^{-1} r\right] \cup \operatorname{ext}\left[\hat{G} \hat{F}^{-1} r\right]$ which extends $\hat{G} \circ \hat{F}^{-1}$ is the composition of the reflection of $[r]$ in a circle interior to it with the diffeomorphism $B$ with the inverse of the reflection of $\left[\hat{G} \hat{F}^{-1} r\right]$ in a circle interior to it. Thus, $\hat{G} \circ \hat{F}^{-1}$ can be extended to a diffeomorphism of $R^{2}$ onto $R^{2}$ and $f \sim g$.

It remains to show that if $f \sim g$, then $\rho(f)=\rho(g)$. If $f \sim g$, then there exist sensepreserving diffeomorphisms $\gamma: S^{1} \rightarrow S^{1}$ and $\Gamma: R^{2} \rightarrow R^{2}$ such that $g=\Gamma \circ f \circ \gamma$. Thus, $g\left(t_{0}\right)$ is on the outer boundary of $g$ iff $f\left(\gamma\left(t_{0}\right)\right)$ is on the outer boundary of $f$; $g\left(x_{i}\right)=g\left(x_{j}\right)$ iff $f\left(\gamma\left(x_{i}\right)\right)=f\left(\gamma\left(x_{j}\right)\right)$ and

$$
\text { sgn det }\left[g^{\prime}\left(x_{i}\right), g^{\prime}\left(x_{j}\right)\right]=\operatorname{sgn} \operatorname{det}\left[f^{\prime}\left(\gamma\left(x_{i}\right)\right), f^{\prime}\left(\gamma\left(x_{j}\right)\right)\right] \text {. }
$$

Thus, if IS $\left(g, t_{0}\right)$ is an intersection sequence of $g$, then it is also an intersection sequence for $f$ with respect to $\gamma\left(t_{0}\right)$ and vice-versa.

Corollary 2.1. If $\rho(f) \cap \rho(g) \neq \varnothing$, then $\rho(f)=\rho(g)$.

Proof. If $\rho(f) \cap \rho(g) \neq \varnothing$, then there is an intersection sequence of $f$ which is also an intersection sequence of $g$. By inspecting the proof of Theorem 2.1, we note that $f$ and $g$ having identical intersection sequences was all that was needed to show that $f \sim g$. Hence, $\rho(f)=\rho(g)$. 
COROllaRY 2.2. Let $n(f)$ denote the number of simple intersection points of $f$. Then $n$ is a numerical invariant.

Proof. Follows directly from the fact that $\rho$ is a complete invariant.

REMARK. A natural question that arises when one considers the intersection sequences of a normal immersion is the question of realizability: Given the set of integers 0 through $2 n+1$, a fixed point free involution * on these integers, and a signing of these integers, $\nu(j)$, such that $\nu(j)=-\nu\left(j^{*}\right)$, is there a normal immersion which has this data for an intersection sequence? Francis [1] has given a constructive algorithm on such data which always answers this question.

3. Maximum and minimum winding numbers. The winding number of a normal immersion $f$ with respect to a point $P$ not on $[f]$, denoted by $w(f, P)$, is defined to be the topological degree of the mapping $(f(t)-P) /(|f(t)-P|)$ of $S^{1}$ into $S^{1}$. The maximum $(w \max )$ and the minimum $(w \min )$ winding numbers of $f$ are defined by

$$
w \max (f)=\max w(f, P), \quad w \min (f)=\min w(f, P)
$$

where the maximum and the minimum are taken over all points $P$ not on $[f]$. For the points $f(t)$ on $[f]$, let $w^{+}(f, f(t))$ and $w^{-}(f, f(t))$ denote the larger and the smaller winding numbers respectively of $f$ about points near $f(t)$ and not on $[f]$. Throughout this section we will assume unless stated otherwise that the domain of $f$ is the interval $[0,2 n+1]$ where $n$ is the number of simple intersection points of $f$.

THEOREM 3.1. Let f be a normal immersion with intersection sequence IS. Let u be a nonintegral parameter value of $f$ with integral part $k$.

(1) If $k \geqq 1$, then

$$
w^{+}(f, f(u))-w^{+}(f, f(0))=w^{-}(f, f(u))-w^{-}(f, f(0))=\sum_{1 \leqq j \leqq k} \nu(j) .
$$

(2) If $k=0$, then

$$
w^{+}(f, f(u))-w^{+}(f, f(0))=w^{-}(f, f(u))-w^{-}(f, f(0))=0 .
$$

The proof of Theorem 3.1 will follow its corollaries and the statement of three lemmas due to Whitney and Titus needed in its proof.

COROLLARY 3.1. Let f be a normal immersion and let IS be an intersection sequence of $f$. Then

$$
w \max (f)=\max _{1 \leqq k \leqq 2 n}\left(\sum_{1 \leqq j \leqq k} \nu(j)\right)+w^{+}(f, f(0))
$$

and

$$
w \min (f)=\min _{1 \leqq k \leqq 2 n}\left(\sum_{1 \leqq j \leqq k} \nu(j)\right)+w^{-}(f, f(0)) .
$$

If $n=0$, then $w \max (f)=w^{+}(f, f(0))$ and $w \min (f)=w^{-}(f, f(0))$. 
Proof. Since the proofs for $w \max (f)$ and $w \min (f)$ are similar, we will consider only the proof for $w \max (f)$. Let $P$ be a point such that $w \max (f)=w(f, P)$ and let $u$ be a parameter value with integral part $k$ such that $f(u)$ is on the boundary of the component of $R^{2}-[f]$ containing $P$. If $n=0$, then $k=0$ and $w \max (f)$ $=w^{+}(f, f(0))$ by Theorem 3.1. If $n>0$, then by Theorem 3.1

$$
w \max (f)=w(f, P)=\sum_{1 \leqq j \leqq k} \nu(j)+w^{+}(f, f(0))
$$

or $w \max (f)=w^{+}(f, f(0))$ according as $k \geqq 1$ or $k=0$ respectively. On the other hand for each integer $l$ in $I S$ such that $1 \leqq l \leqq 2 n$, there exists a point $R$ such that

$$
w(f, R)=\sum_{1 \leqq j \leqq l} \nu(j)+w^{+}(f, f(0)) .
$$

Thus, since

$$
\max _{1 \leqq k \leqq 2 n}\left(\sum_{1 \leqq j \leqq k} v(j)\right) \geqq 0
$$

we have that

$$
w \max (f)=\max _{1 \leqq k \leqq 2 n}\left(\sum_{1 \leqq j \leqq k} \nu(j)\right)+w^{+}(f, f(0)) .
$$

COROLlARY 3.2. $w \max$ and $w$ min are numerical invariants.

Proof. Follows directly from Corollary 3.1 and Theorem 2.1.

Lemma 3.1 (Titus [4, P. 1085], OR WhitNey [6, P. 281]). Let $f$ be a normal immersion and let $I_{1}$ and $I_{2}$ be subintervals of $S^{1}$.

(1) If $\left[f \mid I_{1}\right] \cap\left[f \mid I_{2}\right]=\varnothing$, then there exists an $e>0$ such that whenever $g$ is a normal immersion with $\|f-g\|<e,[F] \cap[G]=\varnothing$ where $F$ is $f \mid I_{1}$ or $g \mid I_{1}$ and $G$ is $f \mid I_{2}$ or $g \mid I_{2}$.

(2) If $\left[f \mid I_{1}\right]$ intersects $\left[f \mid I_{2}\right]$ transversally in exactly one point, then there exists an $e>0$ such that whenever $g$ is a normal immersion with $\|f-g\|<e,[F]$ intersects $[G]$ transversally in exactly one point where $F$ is $f \mid I_{1}$ or $g \mid I_{1}$ and $G$ is $f \mid I_{2}$ or $g \mid I_{2}$. Moreover, if $a, c \in I_{1}$ and $b, d \in I_{2}$ with $f(a)=f(b)$ and $F(c)=G(d)$, then

$$
\text { sgn } \operatorname{det}\left[f^{\prime}(a), f^{\prime}(b)\right]=\operatorname{sgn} \operatorname{det}\left[F^{\prime}(c), G^{\prime}(d)\right] \text {. }
$$

LEMMA 3.2 (TITUS [4, P. 1086]). Given a normal immersion $f$ and an $e>0$, there exists normal immersions $g_{1}$ and $g_{2}$ such that

(1) $\left\|f-g_{i}\right\|<e$ for $i=1$ or 2 ;

(2) $g_{1}$ is on the right of $f\left(i . e ., \operatorname{det}\left[g_{1}-f, f^{\prime}\right]>0\right)$ and $g_{2}$ is on the left of $f(i . e$,, $\left.\operatorname{det}\left[g_{2}-f, f^{\prime}\right]<0\right)$; and

(3) there exists a $d>0$ such that $[f] \cap\left[g_{i}\right]=\varnothing$ on $[t-d, t+d]$ for all $t$ and for $i=1$ or 2 .

LEMMA 3.3 (TiTUs [4, P. 1085]). Let $f$ be a normal immersion and let $h$ be a $C^{1}$-map of $[0,1]$ into $R^{2}$ such that $h(0)$ and $h(1)$ are not on $[f]$ and such that $[h]$ intersects $[f]$ 
transversally in a finite number of points. If $\operatorname{sgn}(j)$ is the sign of the determinant of $f^{\prime}$ with $h^{\prime}$ at the jth point of intersection, then

$$
w(f, h(1))-w(f, h(0))=\sum \operatorname{sgn}(j)
$$

where sum is taken over all intersection points of $[f]$ with $[h]$.

Proof of Theorem 3.1. Partition the interval $[0, u]$ into a finite number of closed segments so that each positive integer in the interval $[0, u]$ is in the interior of exactly one segment. Let $I$ be a segment of this partition with $I^{c}=[0,2 n+1]-I$. If $I$ contains the positive integer $j$, then $[f \mid I]$ intersects $\left[f \mid I^{c}\right]$ transversally in the point $f(j)=f\left(j^{*}\right)$. If $I$ does not contain a positive integer, then $[f \mid I] \cap\left[f \mid I^{c}\right]=\varnothing$. Thus, by Lemma 3.1 parts (1) and (2), each segment of the partition determines an $e>0$. Let $e_{1}$ be the minimum of the finite number of $e$ 's determined in this way. With $e_{1}$ replacing the $e$ of Lemma 3.2, we let $g$ denote either of the normal immersions $g_{1}$ or $g_{2}$ and if necessary we subdivide the segments of the partition so that each has length less than $2 d$ where $g_{1}, g_{2}$, and $d$ are given in Lemma 3.2.

If $I$ is the segment of the partition containing the positive integer $j$, then $[f \mid I]$ intersects $\left[f \mid I^{c}\right]$ transversally in the point $f(j)=f\left(j^{*}\right)$. Thus, by Lemma 3.1 part (2), $[g \mid I]$ intersects $\left[f \mid I^{c}\right]$ transversally in a point, $g(c)=f(d)$, with sgn det $\left[f^{\prime}(d), g^{\prime}(c)\right]$ $=\operatorname{sgn} \operatorname{det}\left[f^{\prime}\left(j^{*}\right), f^{\prime}(j)\right]=\nu(j)$. Moreover, from Lemma 3.2 part (3), we have that $[g \mid I] \cap[f \mid I]=\varnothing$. Thus, $[g \mid I]$ intersects $[f]$ transversally in exactly one point with the sign of the determinant of $f^{\prime}$ with $g^{\prime}$ at this point being $\nu(j)$. If $I$ is a segment of the partition which does not contain a positive integer, then $[f \mid I] \cap\left[f \mid I^{c}\right]=\varnothing$. By Lemma 3.1 part (1) and Lemma 3.2 part (3), we have that $[g \mid I] \cap[f]=\varnothing$. Therefore, $[g \mid[0, u]]$ intersects $[f]$ in exactly $k$ points, where $k$ is the integral part of $u$, with the sign of the determinant of $f^{\prime}$ with $g^{\prime}$ at the $j$ th point being $\nu(j)$. Thus, by Lemma 3.3,

$$
w(f, g(u))-w(f, g(0))=\sum_{1 \leqq j \leqq k} \nu(j) .
$$

Into this equation substitute first $g_{1}$ and then $g_{2}$ for $g$, equate left-hand sides, and rearrange to obtain

$$
w\left(f, g_{1}(u)\right)-w\left(f, g_{2}(u)\right)=w\left(f, g_{1}(0)\right)-w\left(f, g_{2}(0)\right)
$$

From this equation and the fact that $g_{1}$ and $g_{2}$ are on opposite sides of $f$, it follows that if $w^{+}(f, f(u))=w\left(f, g_{1}(u)\right)$, then $w^{+}(f, f(0))=w\left(f, g_{1}(0)\right)$ with $w^{-}(f, f(u))=$ $w\left(f, g_{2}(u)\right)$ and $w^{-}(f, f(0))=w\left(f, g_{2}(0)\right)$. Substituting these relations into equation (1) gives the desired result. If $w^{+}(f, f(u))=w\left(f, g_{2}(u)\right)$, interchange $g_{1}$ and $g_{2}$ in the above relations and substitute in equation (1) to obtain the desired result.

4. Order. The order of a normal immersion $f$, denoted by $O(f)$, is defined to be the maximum taken over all lines $L$ in $R^{2}$ of the number of times that $L$ intersects $[f]$.

THEOREM 4.1. $O(f) \geqq 2(w \max (f)-w \min (f))$. 
Proof. Let $P$ and $Q$ be points such that $w \max (f)=w(f, P)$ and $w \min (f)$ $=w(f, Q)$. Let $L$ be the line joining $P$ and $Q$ and let $R$ and $S$ be points in the unbounded component of $R^{2}-[f]$ with $R P Q S$ being the order of the points on $L$. If $L$ intersects $[f]$ in an infinite number of points, then the inequality is satisfied since $w(f, P)$ and $w(f, Q)$ are finite. If $L$ is a tangent line to $[f]$ at some point, an arc of $[f]$ about that point can be replaced by another arc which does not have $L$ as a tangent line and which has no more intersection points with $L$ than the original arc. Thus, we assume that $L$ intersects $[f]$ transversally in a finite number of points. We now apply Lemma 3.3 with $h$ being a representation of the segments $R P, P Q$, and $Q S$ successively to obtain:

(1) $R P$ intersects $[f]$ in at least $w \max (f)$ points,

(2) $P Q$ intersects $[f]$ in at least $w \max (f)-w \min (f)$ points, and

(3) $Q S$ intersects $[f]$ in at least $-w \min (f)$ points.

Therefore, $L$ intersects $[f]$ in at least $2(w \max (f)-w \min (f))$ points.

It is easily seen that the order of a normal immersion is not a numerical invariant. To define a numerical invariant in terms of the order of a normal immersion, let $D$ be an equivalence class of diffeomorphically equivalent normal immersions and define the order of $D$ by

$$
O(D)=\min _{f \in D} O(f)
$$

Corollary 4.1. $O(D) \geqq 2(w \max (D)-w \min (D))$.

Proof. Follows directly from Corollary 3.2 and Theorem 4.1.

REmark. The inequalities in Theorem 4.1 and Corollary 4.1 are the best possible as seen by the figure eight and its associated equivalence class.

COROLlaRY 4.2. Let $f$ be a complex polynomial of degree $m$ such that $f \mid S^{1}$ is an immersion. Then $w \max \left(f \mid S^{1}\right) \leqq m$.

Proof. From the density property of normal immersions we assume that $f \mid S^{\mathbf{1}}$ is a normal immersion. By Theorem 4.1 and the fact that $f$ is analytic we have that $2 w \max \left(f \mid S^{1}\right) \leqq O\left(f \mid S^{1}\right)$. But $f$ being a polynomial of degree $m$ implies that $O\left(f \mid S^{1}\right) \leqq 2 m$. Thus, $w \max \left(f \mid S^{1}\right) \leqq m$.

5. Subloops. Because normal immersions have a finite number of simple intersection points, many statements can be proved using finite induction. To use the induction hypothesis one "cuts" the normal immersion at one of its simple intersection points to obtain after modification two normal immersions each with less simple intersection points than the original. We use this technique in studying the tangent winding number and the total variation of a normal immersion in $\$ \$ 6$ and 7. The purpose of this section is to define and establish results for subloops of a normal immersion. 
Let $f$ be a normal immersion with $n(f)>0$ and let $I S$ be an intersection sequence of $f$. For each positive integer $k<k^{*}$ in $I S$ define two mappings $f_{k}$ and $f / k$ by

$$
f_{k}(t)=f(t+k), \quad 0 \leqq t \leqq k^{*}-k,
$$

and

$$
\begin{aligned}
(f \mid k)(t) & =f(t), & & 0 \leqq t \leqq k, \\
& =f\left(t+k^{*}-k\right), & & k \leqq t \leqq 2 n+1-k^{*}+k .
\end{aligned}
$$

The mapping $f_{k}$ is called the subloop of $f$ determined by $k$ and the mapping $f / k$ is called the complementary subloop of $f_{k}$. Let $\tilde{f}_{k}$ and $\tilde{f} / k$ denote the normal immersions obtained from $f_{k}$ and $f / k$ respectively by smoothing their corners at $f_{k}(0)$ $=(f / k)(k)$ and taking the parameter domain to be $S^{1}$.

THEOREM 5.1. Let $f$ be a normal immersion with $n(f)>0$ and with intersection sequence IS. Let $k<k^{*}$ be a positive integer in IS and let $u$ and $v$ be parameter values such that $k-1<v<k$ and $k^{*}<u<k^{*}+1$. Then

$$
w\left(f_{k}, f(v)\right)=w\left(f_{k}, f(u)\right)=\sum_{j L k} v(j)=\sum_{j R k} \nu(j) .
$$

If one of the sets $\{j \mid j L k\}$ or $\{j \mid j R k\}$ is empty, set $\sum \nu(j)$ for that set equal to zero.

Proof. Since $[f \mid[0, v]]$ intersects $\left[\tilde{f}_{k}\right]$ in the points $f(j)=f\left(j^{*}\right)$ where $j L k$ with

$$
\operatorname{sgn} \operatorname{det}\left[\tilde{f}_{k}^{\prime}\left(j^{*}\right),(f \mid[0, v])^{\prime}(j)\right]=\operatorname{sgn} \operatorname{det}\left[f^{\prime}\left(j^{*}\right), f^{\prime}(j)\right]=\nu(j),
$$

we have by Lemma 3.3 that

$$
w\left(\tilde{f}_{k}, f(v)\right)-w\left(\tilde{f}_{k}, f(0)\right)=\sum_{j L k} v(j) .
$$

Since $f(0)$ is on the outer boundary of $f$ and $w\left(\tilde{f}_{k}, f(v)\right)=w\left(f_{k}, f(v)\right)$, we have $w\left(f_{k}, f(v)\right)=\sum_{j L k} \nu(j)$. In a similar manner, replacing $f \mid[0, v]$ by $f \mid[u, 2 n+1]$, we have $w\left(f_{k}, f(u)\right)=\sum_{j R k} \nu(j)$. Finally, since $f(u)$ and $f(v)$ are in the same component of $R^{2}-\left[f_{k}\right]$, we have that $w\left(f_{k}, f(u)\right)=w\left(f_{k}, f(v)\right)$.

COROLlaRY 5.1. If $f$ is a normal immersion with intersection sequence IS and $k<k^{*}$ is an integer in IS, then $\sum_{k<j<k^{*}} \nu(j)=0$.

Proof. Let $j$ be an integer such that $k<j<k^{*}$. If $k<j<j^{*}<k^{*}$ or $k<j^{*}<j<k^{*}$, then both $\nu(j)$ and $\nu\left(j^{*}\right)$ are summands with $\nu(j)+\nu\left(j^{*}\right)=0$. Thus, it remains to show that

$$
\sum_{j^{*}<k<j<k^{*}} \nu(j)+\sum_{k<j<k^{*}<j^{*}} \nu(j)=0 .
$$

But this is equivalent to showing that

$$
-\left(\sum_{j \cdot L k} \nu(j)\right)+\sum_{j R k} \nu(j)=0
$$

This follows from Theorem 5.1. 
We now restrict our attention to simple subloops of a normal immersion $f$ with $n(f)>0$. That is, we consider the positive integers $k<k^{*}$ in an intersection sequence of $f$ with the property that $\{j \mid j \subset k\}=\varnothing$. Since for each such positive integer $k$, $\left[f_{k}\right]$ is an oriented Jordan curve, we have from Theorem 5.1 that $\sum_{j L k} \nu(j)$ is $-1,0$, or 1. A simple subloop $f_{k}$ of $f$ is said to be

(a) positive exterior iff $\sum_{j L k} \nu(j)=0$ and $\nu(k)=+1$,

(b) negative exterior iff $\sum_{j L k} \nu(j)=0$ and $\nu(k)=-1$,

(c) positive interior iff $\sum_{j k} \nu(j)=+1$, and

(d) negative interior iff $\sum_{j L k} \nu(j)=-1$.

Note that these definitions are relative to an intersection sequence $I S$ of the normal immersion $f$.

REMARK. Every nonsimple normal immersion has exterior simple subloops since the smallest positive integer $k$ in an intersection sequence such that $f_{k}$ is simple has the property that the relation $j L k$ is empty. Thus, if an intersection sequence $I S$ of a normal immersion $f$ is properly nested (i.e., the relation $j L k$ is empty for all $k$ ), every simple subloop is exterior. Even if an intersection sequence is not properly nested, it does not necessarily imply that there will be interior simple subloops.

COROLlaRY 5.2. Let $f$ be a normal immersion with intersection sequence IS. If $f_{k}$ is a negative [positive] interior simple subloop of $f$, then $\nu(k)=+1[\nu(k)=-1]$.

Proof. Since the proof for $f_{k}$ being a positive interior simple subloop is similar to the proof for $f_{k}$ being a negative interior simple subloop, we will consider only the case where $f_{k}$ is a negative interior simple subloop. Let $u$ and $v$ be parameter values such that $k-1<u<k<v<k+1$. From Theorem 3.1, we have

$$
w^{+}(f, f(v))-w^{+}(f, f(0))=\sum_{1 \leqq j \leqq k} v(j)
$$

and

$$
w^{+}(f, f(u))-w^{+}(f, f(0))=\sum_{1 \leqq j \leqq k-1} \nu(j) .
$$

Subtracting the second equation from the first we have

$$
w^{+}(f, f(v))-w^{+}(f, f(u))=v(k)= \pm 1 .
$$

Since $\sum_{j L k} \nu(j)=-1$, we have by Theorem 5.1 that $w\left(f_{k}, f(u)\right)=-1$. Thus, $\left[f_{k}\right]$ is a negatively oriented Jordan curve and a point $P$ near $f(v)$ such that $w^{+}(f, f(v))$ $=w(f, P)$ is in the exterior of $\left[f_{k}\right]$. Also, $f(u)$ and the points $Q$ near $f(u)$ such that $w^{+}(f, f(u))=w(f, Q)$ are in the interior of $\left[f_{k}\right]$. Hence, $w(f, P)>w(f, Q)$ and $\nu(k)=w^{+}(f, f(v))-w^{+}(f, f(u))=w(f, P)-w(f, Q)=+1$.

COROLlaRY 5.3. Let $f$ be a normal immersion with intersection sequence IS and let $f_{k}$ be a simple subloop of $f$. Let $\varphi_{k}$ be the oriented angle from $f^{\prime}\left(k^{*}\right)$ to $f^{\prime}(k)$ and let $\psi_{k}$ be the change in a continuous branch of $\arg f^{\prime}(t)$ on the interval $\left[k, k^{*}\right]$. If $f_{k}$ 
is a positive or negative interior simple subloop, then $\left|\psi_{k}\right|=2 \pi+\left|\varphi_{k}\right|$. If $f_{k}$ is a positive or negative exterior simple subloop, then $\left|\psi_{k}\right|=2 \pi-\left|\varphi_{k}\right|$.

Proof. If $f_{k}$ is a positive interior simple subloop, then $\left[f_{k}\right]$ is a positively oriented Jordan curve and $\psi_{k}+\varphi_{k}=2 \pi$. But the angle $\varphi_{k}$ is negative since $\nu(k)=-1$ from Corollary 5.2. Hence, $\psi_{k}=2 \pi+\left|\varphi_{k}\right|$. If $f_{k}$ is a negative interior simple subloop, then $\left[f_{k}\right]$ is a negatively oriented Jordan curve and $\psi_{k}+\varphi_{k}=-2 \pi$. In this case $\nu(k)=+1$ and $\varphi_{k}$ is positive. Hence, $\psi_{k}=-\left(2 \pi+\left|\varphi_{k}\right|\right)$. The results for $f_{k}$ being a positive or negative exterior simple subloop follow in a similar fashion.

COROLLARY 5.4. Let $f$ be a normal immersion with intersection sequence IS. If all the simple subloops of $f$ determined by IS are positive exterior and there exist at least two simple subloops, then there exist two disjoint simple subloops (i.e., their image curves have no points in common).

Proof. Let $i$ and $j$ be the first two integer parameter values such that $f_{i}$ and $f_{j}$ are simple subloops. Assuming $i<j$, we have either $i<i^{*}<j<j^{*}$ or $i<j<i^{*}<j^{*}$. In the first case, $\left[f_{i}\right] \cap\left[f_{j}\right]=\varnothing$; for, if not, there exists an integer $k$ such that $i<k<i^{*}<j<k^{*}<j^{*}$ which contradicts the fact that $j$ is the second integer such that $f_{j}$ is simple.

We will now show that the case $i<j<i^{*}<j^{*}$ is impossible. Let $u$ and $v$ be parameter values such that $j^{*}-1<u<j^{*}<v<j^{*}+1$ and let $P=f(v)$ and $Q=f(u)$. Since $f_{i}$ and $f_{j}$ are positive exterior, $\nu(i)=\nu(j)=+1$. Thus, replacing the mapping $h$ in Lemma 3.3 by $f \mid[u, v]$ and the normal immersion $f$ by $f_{i}$, we have

$$
w\left(f_{i}, P\right)-w\left(f_{i}, Q\right)=v\left(j^{*}\right)=-1
$$

Thus, $P$ is in the exterior and $Q$ is in the interior of $\left[f_{i}\right]$. Now let $r$ be a parameter value such that $i^{*}<r<i^{*}+1$. Since $f_{i}$ is a positive exterior simple subloop, we have by Theorem 5.1 that the point $R=f(r)$ is in the exterior of $\left[f_{i}\right]$. Thus, since $i^{*}<r<u<j^{*}$ with $f(r)$ in the exterior and $f(u)$ in the interior, there is an integer parameter value $k$ such that $i<k<i^{*}<r<k^{*}<u<j^{*}$. If $i<k<j<i^{*}<k^{*}<j^{*}$, then $j$ is not the second parameter value such that $f_{j}$ is a simple subloop. If $i<j<k<i^{*}<k^{*}<j^{*}$, then $f_{j}$ is not a simple subloop. Hence, it is impossible that $i<j<i^{*}<j^{*}$.

6. Tangent winding number. The tangent winding number $\tau$ of a normal immersion $f$ is defined to be the topological degree of the Gauss map $f^{\prime}(t) /\left|f^{\prime}(t)\right|$ of $S^{1}$ into $S^{1}$. In Theorem 6.1 we give a proof based on the ideas developed in $\S 5$ of a result due to Whitney [6] which computes $\tau(f)$ from an intersection sequence of $f$. As a corollary we obtain the fact that $\tau$ is a numerical invariant. Next we obtain a result which computes the tangent winding number of a subloop using the intersection sequence which determines that subloop. Finally we obtain the inequality $n(f) \geqq 2 w \max (f)-\tau(f)-1$. 
LEMMA 6.1. Let $f$ be a normal immersion with $n(f)>0$ and with intersection sequence IS. If $k$ is a positive integer in IS with $k<k^{*}$, then $\tau\left(\tilde{f}_{k}\right)+\tau(\tilde{f} \mid k)=\tau(f)$.

Proof. Let $\operatorname{Arg} f^{\prime}(t)$ denote a continuous branch of $\arg f^{\prime}(t)$ and let $\Delta_{a, b} \operatorname{Arg} f^{\prime}(t)$ denote the change in $\operatorname{Arg} f^{\prime}(t)$ from $a$ to $b$. Let $\varphi_{k}$ be the oriented angle from $f^{\prime}\left(k^{*}\right)$ to $f^{\prime}(k)$. Then

$$
2 \pi \tau\left(\tilde{f}_{k}\right)=\varphi_{k}+\Delta_{k, k^{*}} \operatorname{Arg} f^{\prime}(t)
$$

and

$$
2 \pi \tau(\tilde{f} \mid k)=\Delta_{0, k} \operatorname{Arg} f^{\prime}(t)+\left(-\varphi_{k}\right)+\Delta_{k^{*}, 2 n+1} \operatorname{Arg} f^{\prime}(t) .
$$

Thus, $2 \pi \tau\left(\tilde{f}_{k}\right)+2 \pi \tau(\tilde{f} / k)=2 \pi \tau(f)$.

THEOREM 6.1. If $f$ is a normal immersion and IS $\left(f, t_{0}\right)$ is an intersection sequence of $f$, then $\tau(f)=\sum_{j<j} \nu(j)$.

Proof. The proof will be by induction on $n(f)$. If $n(f)=0$, then $\tau(f)$ is +1 or -1 according as $[f]$ is a positively or negatively oriented Jordan curve. But the orientation of $[f]$ is given by $\nu(0)$. If $\nu(0)=+1$, then $w^{+}(f, f(0))=+1$ and $[f]$ is positively oriented. If $\nu(0)=-1$, then $w^{+}(f, f(0))=0$ and $[f]$ is negatively oriented.

If $n(f)>0$, let $k$ be the first integer such that $f_{k}$ is a simple subloop. Let $u$ and $v$ be parameter values such that $k-1<u<k<k^{*}<v<k^{*}+1$. By Theorem 5.1 and the fact that $\{j \mid j L k\}=\varnothing$, we have

$$
w\left(f_{k}, f(u)\right)=w\left(f_{k}, f(v)\right)=\sum_{j L k} \nu(j)=\sum_{j R k} \nu(j)=0 .
$$

From this it follows that $f(u)$ and $f(v)$ are in the unbounded component of $R^{2}-\left[f_{k}\right]$ and that $\left[f_{k}\right]$ is positively or negatively oriented according as $\nu(k)$ is +1 or -1 respectively. Thus, $\tau\left(\tilde{f}_{k}\right)=\nu(k)$. The normal immersion $\tilde{f} / k$ has an intersection sequence $\operatorname{IS}\left(f / k, t_{0}\right)$ which is equivalent to the subset $\operatorname{SIS}\left(f, t_{0}\right)$ of $\operatorname{IS}\left(f, t_{0}\right)$ obtained by deleting the integers $j$ and $j^{*}$ such that $j L k, j R k$, or $j=k$ (i.e., there is a 1-1 correspondence between the integers in IS $\left(\tilde{f} \mid k, t_{0}\right)$ and the integers in SIS $\left(f, t_{0}\right)$ which preserves order, the involution *, and the signing of the integers). Thus, by the induction hypothesis, we have

$$
\tau(\tilde{f} \mid k)=\sum \nu(j) \quad\left(j \in \operatorname{SIS}\left(f, t_{0}\right) \text { and } j<j^{*}\right) .
$$

By Lemma 6.1 and the fact that $\sum_{j L k} \nu(j)=\sum_{j R k} \nu(j)=0$, we have

$$
\begin{aligned}
\tau(f) & =\tau\left(\tilde{f_{k}}\right)+\tau(\tilde{f} \mid k) \\
& =\nu(k)+\sum_{j \in S I S} \nu(j)+\sum_{j L k} \nu(j)+\sum_{j R k} \nu(j) \\
& =\sum \nu(j) \quad\left(j \in \operatorname{IS~}\left(f, t_{0}\right) \text { and } j<j^{*}\right) .
\end{aligned}
$$

COROllary 6.1. $\tau$ is a numerical invariant. 
Proof. Follows directly from Theorems 6.1 and 2.1.

REMARK. It is easily seen that $\tau$ is not a complete invariant. However, $\tau$ is a complete invariant for regular homotopy as shown by the Whitney-Graustein Theorem [6]. Francis [2] has shown that if $\tau(f)=\tau(g)$, then there exists an immersion with one fold defined on an annulus such that its restriction to the two boundaries of the annulus are $f$ and $g$ respectively.

COROLlaRY 6.2. Let $f$ be a normal immersion with $n(f)>0$ and let $\operatorname{IS}\left(f, t_{0}\right)$ be an intersection sequence of $f$. For each positive integer $k<k^{*}$,

$$
\tau\left(\tilde{f}_{k}\right)=\nu(k)+\sum_{j \in k} \nu(j)+2 \sum_{j L k} \nu(j)
$$

Proof. As in the proof of Theorem 6.1, we obtain a subset SIS $\left(f, t_{0}\right)$ of IS $\left(f, t_{0}\right)$ equivalent to the intersection sequence IS $\left(\tilde{f} / k, t_{0}\right)$ by deleting the integers $j$ and $j^{*}$ such that $j=k, j L k, j R k$, or $j \subset k$. From Theorem 6.1, we have

$$
\tau(f)=\sum \nu(j) \quad\left(j \in \operatorname{ISS}\left(f, t_{0}\right) \text { and } j<j^{*}\right)
$$

and

$$
\tau(\tilde{f} \mid k)=\sum \nu(j) \quad\left(j \in \operatorname{SIS}\left(f, t_{0}\right) \text { and } j<j^{*}\right) .
$$

From these two equations and Lemma 6.1, we have

$$
\tau\left(\tilde{f_{k}}\right)=\tau(f)-\tau(\tilde{f} \mid k)=\nu(k)+\sum_{j \subset k} \nu(j)+2 \sum_{j L k} \nu(j) .
$$

THEOREM 6.2. If $f$ is a normal immersion, then $n(f) \geqq 2 w \max (f)-\tau(f)-1$.

Proof. The proof will be by induction on $n(f)$. If $n(f)=0$, then $w \max (f)$ is +1 or 0 according as $\tau(f)$ is +1 or -1 respectively. In either case the inequality is satisfied.

If $n(f)>0$, let $k$ be the first integer in an intersection sequence $I S$ of $f$ such that $f_{k}$ is a simple subloop. Since $\{j \mid j L k\}=\varnothing, f_{k}$ is either a positive or negative exterior simple subloop of $f$. If $f_{k}$ is a positive exterior simple subloop, then $\tau(f)=\tau(\tilde{f} \mid k)+1$ and $n(f) \geqq n(\tilde{f} / k)+1$. Moreover, if $P$ is a point such that $w(f, P)=w \max (f)$, then

$$
w \max (f)=w(f, P)=w\left(f_{k}, P\right)+w(f \mid k, P) \leqq w \max (f / k)+1 .
$$

From these inequalities and the induction hypothesis, we have

$$
n(f) \geqq n(\tilde{f} \mid k)+1 \geqq 2 w \max (\tilde{f} / k)-\tau(\tilde{f} / k) \geqq 2 w \max (f)-\tau(f)-1 .
$$

Similarly, if $f_{k}$ is a negative exterior simple subloop, we obtain the inequalities $\tau(f)=\tau(\tilde{f} \mid k)-1, n(f) \geqq n(\tilde{f} \mid k)+1$, and $w \max (f) \leqq w \max (\tilde{f} / k)$. These inequalities together with the induction hypothesis yield the desired result.

REMARK. It can be shown that if the inequality in Theorem 6.2 is an equality for a normal immersion $f$, then every intersection sequence of $f$ is properly nested 
(i.e., the relations $j L k$ and $j R k$ are empty for all $k$ ). Moreover, for each nonnegative integer $m$ there is a normal immersion $f$ such that $n(f)=m$ and for which the inequality of Theorem 6.2 is an equality.

7. Total variation. The total variation $v$ of a normal immersion $f$ is defined to be the length of the Gauss map $f^{\prime}(t) /\left|f^{\prime}(t)\right|$ on $S^{1}$. The purpose of this section is to demonstrate the following theorem and to obtain necessary conditions that a normal immersion be simple.

THEOREM 7.1. If $f$ is a normal immersion, then $w \max (f) \leqq 1 / 2 \pi \cdot v(f)$ with strict inequality if $f$ has inflection points.

Proof. The proof will be by induction on $n(f)$. If $n(f)=0$, then $w \max (f)$ $\leqq|\tau(f)| \leqq(1 / 2 \pi) v(f)$ with strict inequality if $f$ has inflection points. If $n(f)>0$ and $I S$ is an intersection sequence of $f$, let $v_{j, k}(f)$ denote the total variation of $f$ on the interval $[j, k]$. Let $\varphi_{k}$ and $\psi_{k}$ be as defined in Corollary 5.3. We consider three cases.

Case 1. There exists a positive integer $k<k^{*}$ in $I S$ such that $f_{k}$ is either a positive or negative interior simple subloop of $f$. Thus, by Corollary 5.3, we have $v_{k, k^{*}}(f)$ $\geqq\left|\psi_{k}\right|=2 \pi+\left|\varphi_{k}\right|$. This together with the fact that $v(\tilde{f} \mid k)=v_{0, k}(f)+\left|\varphi_{k}\right|+v_{k^{*}, 2 n+1}(f)$ yields the result that $v(\tilde{f} \mid k)+2 \pi \leqq v(f)$. Now, as in the proof of Theorem 6.2 , we have

$$
w \max (f) \leqq w \max (\tilde{f} \mid k)+1 .
$$

From the last two inequalities and the induction hypothesis we have

$$
w \max (f) \leqq w \max (\tilde{f} / k)+1 \leqq(1 / 2 \pi) v(\tilde{f} \mid k)+1 \leqq(1 / 2 \pi) v(f) .
$$

If $f$ has inflection points, then either $w \max (\tilde{f} \mid k)<(1 / 2 \pi) v(\tilde{f} \mid k)$ or $v_{k, k^{*}}(f)>\left|\psi_{k}\right|$.

Case 2. There exists a positive integer $k<k^{*}$ in $I S$ such that $f_{k}$ is a negative exterior simple subloop. By Corollary 5.3 and the fact that $0<\left|\varphi_{k}\right|<\pi$, we have $\left|\psi_{k}\right|>\left|\varphi_{k}\right|$. By an argument similar to that used in Case 1 , we have $v(\tilde{f} \mid k)<v(f)$. This together with the induction hypothesis and the fact that $w \max (f) \leqq w \max (f / k)$, yields the result $w \max (f)<(1 / 2 \pi) v(f)$.

Case 3. All simple subloops of $f$ are positive exterior. If $f$ has at least two simple subloops, then there exists two disjoint simple subloops by Corollary 5.4. Thus, there exists a positive integer $k<k^{*}$ in $I S$ such that $w \max (f)=w \max (f / k)$. From this and the induction hypothesis, we have $w \max (f)<(1 / 2 \pi) v(f)$. Assume that $f$ has exactly one simple subloop $f_{k}$. Let $u$ be a parameter value such that $k<u<k+1$. By Theorem 3.4,

$$
w^{+}(f, f(u))-w^{+}(f, f(0))=\sum_{1 \leqq j \leqq k} \nu(j) .
$$

Since $f_{k}$ is the only simple subloop of $f$, we have that $k^{*}=k+1$ and that $j=k$ or $k \subset j$ for all integers $j<j^{*}$ in IS. From Theorem 6.1 and Corollary 6.2, we have

$$
\tau(f)=\sum_{j<j^{*}} \nu(j)=\sum_{1 \leqq j \leqq k} \nu(j)+\nu(0)=\tau\left(\tilde{f}_{1}\right)+\nu(0) .
$$


If $w \max (f)=w^{+}(f, f(u))$ and $\nu(0)=+1$, then

$$
\begin{aligned}
w \max (f) & \leqq \sum_{1 \leqq j \leqq k} \nu(j)+1 \text { from }(1) \\
& \leqq \tau(f) \text { from }(2) \\
& \leqq(1 / 2 \pi) v(f)
\end{aligned}
$$

with strict inequality if $f$ has inflection points. If $w \max (f)=w^{+}(f, f(u))$ and $\nu(0)=-1$, then

$$
\begin{aligned}
w \max (f) & \leqq \sum_{1 \leqq j \leqq k} \nu(j) \quad \text { from }(1) \\
& \leqq \tau\left(\tilde{f}_{1}\right) \quad \text { from }(2) \\
& \leqq(1 / 2 \pi) v\left(\tilde{f}_{1}\right) \leqq(1 / 2 \pi) v(f) .
\end{aligned}
$$

If $w \max (f)>w^{+}(f, f(u))$, then

$$
w \max (f)=w \max (\tilde{f} \mid k) \leqq(1 / 2 \pi) v(\tilde{f} \mid k)<(1 / 2 \pi) v(f) .
$$

REMARK. As a corollary of this result one obtains the function theoretic result due to Umezawa [5] that if $F(z)$ is meromorphic on $|z| \leqq 1$ and $F^{\prime}(z) \neq 0$ on $S^{1}$, then the number of preimages of a point is less than or equal to $(1 / 2 \pi) v\left(F \mid S^{1}\right)$ plus the number of poles of $F$.

To obtain a numerical invariant, let $D$ be an equivalence class of diffeomorphically equivalent normal immersions and define the total variation $v$ of $D$ by $v(D)=$ $\inf _{f \in D} v(f)$.

COROLlaRY 7.1. $w \max (D) \leqq(1 / 2 \pi) v(D)$.

Proof. Follows directly from Corollary 3.2 and Theorem 7.1.

REMARK. The figure eight and its associated equivalence class is an example of a situation where one has strict inequality in Theorem 7.1 for each element of the equivalence class and equality in Corollary 7.1. Also, it seems geometrically clear, particularly in the cases where the intersection sequences are properly nested, that $v(D)$ is a multiple of $\pi$.

COROLlaRY 7.2. If $f$ is a normal immersion, then

(1) $|w(f, P)| \leqq(1 / 2 \pi) v(f)$ for all points $P$ not on $[f]$ and

(2) $v(f) \geqq 2 \pi$ with strict inequality if $n(f)>0$.

Proof. In order to show that $|w(f, P)| \leqq(1 / 2 \pi) v(f)$ for all points $P$ not on $[f]$, it suffices to show that $|w \min (f)| \leqq(1 / 2 \pi) v(f)$. This inequality is obtained by reversing the orientation of $f$ and applying Theorem 7.1.

The fact that $v(f) \geqq 2 \pi$ follows directly from the fact that $|w(f, P)| \leqq(1 / 2 \pi) v(f)$ for all points $P$ not on $[f]$. To obtain strict inequality when $n(f)>0$, we use the fact that the total variation of $f$ on a simple subloop is greater than $\pi$. If $f$ has two simple subloops, then the sum of the total variation on each is greater than $2 \pi$. 
If $f$ has one simple subloop, then the sum of the total variation of $f$ on it and the total variation of $f$ on the interval $\left[1^{*}, 2 n+1\right] \cup[0,1]$ is greater than $2 \pi$.

COROLlary 7.3. If $f$ is a normal immersion with $|\tau(f)| \leqq 1$ and no inflection points, then $f$ is simple.

Proof. Since $f$ has no inflection points, $v(f)=2 \pi|\tau(f)| \leqq 2 \pi$. Thus, by Corollary 7.2 part $(2), v(f)=2 \pi$ and $n(f)=0$.

COROLlaRY 7.4. If $f$ is a normal immersion with $\tau(f)=1, v(f) \leqq 4 \pi$, and $w(f, P) \geqq 0$ for all $P$ not on $[f]$, then $f$ is simple.

Proof. Assume $n(f)>0$ and let $I S$ be an intersection sequence of $f$. By Corollary 3.1 and the fact that $w(f, P) \geqq 0$ for all points $P$ not on $[f]$, we have that $\nu(0)=\nu(1)$ $=+1$ and that $w \max (f) \geqq 2$. By Theorem 7.1 and the fact that $\tau(f)=1$, we obtain the fact that $\nu(f) \neq 2 \pi \cdot \tau(f)$ and thus that $f$ has inflection points. But then by Theorem 7.1 , we have that $v(f)>4 \pi$ which contradicts the hypothesis that $v(f) \leqq 4 \pi$.

REMARK. The following three curves show the necessity of each of the hypothesis of Corollary 7.4 .
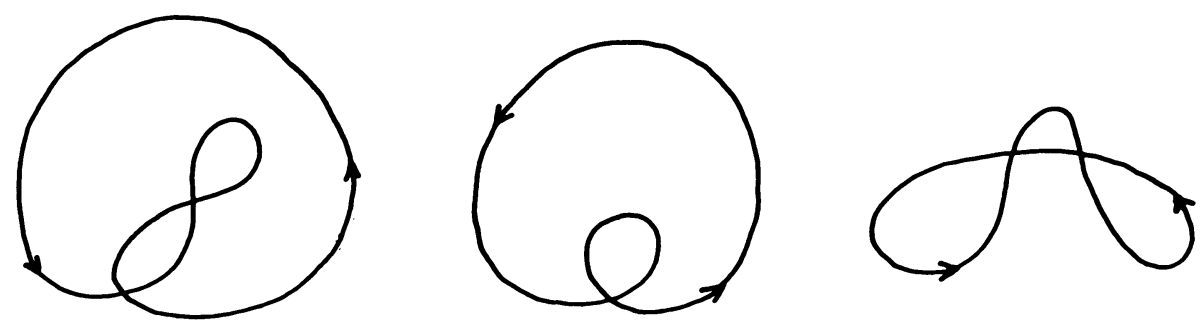

\section{BIBLIOGRAPHY}

1. G. K. Francis, Null genus realizability criterion for abstract intersection sequences, J. Combinatorial Theory 7 (1969), 331-341. MR 40 \#5475.

2. - - The folded ribbon theorem. A contribution to the study of immersed circles, Trans. Amer. Math. Soc. 141 (1969), 271-303. MR 39 \#4863.

3. Charles Loewner, $A$ topological characterization of a class of integral operators, Ann. of Math. (2) 49 (1948), 316-332. MR 9, 502.

4. C. J. Titus, $A$ theory of normal curves and some applications, Pacific J. Math. 10 (1960), 1083-1096. MR 22 \#5014.

5. T. Umezawa, On the theory of univalent functions, Tôhoku Math. J. (2) 7 (1955), 212-228. MR 17, 1068.

6. Hassler Whitney, On regular closed curves in the plane, Compositio Math. 4 (1937), 276-284.

Department of Mathematics, University of Michigan, Dearborn, Michigan 48128 